Artigo original

Hegemonia - Revista Eletrônica do Programa de Mestrado em Direitos Humanos, Cidadania e Violência/Ciência Política do Centro Universitário Unieuro

ISSN: 1809-1261

UNIEURO, Brasília, número 23, Janeiro a Junho de 2018, pp. 78-93.

Recebido em: 15/11/2017

Avaliado em: 9/12/2017

Aprovado em: 18/12/2017

\title{
PREVALÊNCIA DE SOBREPESO/OBESIDADE EM CRIANÇAS E ADOLESCENTES DE UMA ESCOLA PÚBLICA DO DISTRITO FEDERAL:
}

\author{
Estudo de caso
}

\begin{abstract}
Patrícia Borel de Sousa, ${ }^{1}$ Kelb Bousquet Santos, ${ }^{2}$ Marcos Takashi Obara, ${ }^{3}$ Roberta Gomes Carvalho, ${ }^{4}$ Aldira Duarte Domiguez, ${ }^{5}$ e Vanessa Resende Nogueira Cruvinel ${ }^{6}$
\end{abstract}

RESUMO: Objetivo: Identificar o perfil antropométrico e a prevalência de sobrepeso e obesidade em crianças e adolescentes de uma escola pública da Ceilândia - DF no período de 2014 e 2015. Métodos: Estudo epidemiológico do tipo transversal com abordagem descritiva de dados antropométricos de 531escolares do período vespertino da Escola Classe 48 para analisar as seguintes variáveis: perfil do estado antropométrico segundo o ano, sexo e faixa etária. Resultados:A prevalência do excesso de peso dos escolares analisados foi de 34,5\% no ano de 2014 (Sexo masculino: 17,5\%.Sexo feminino: 17,1\%). Já no ano de 2015 foi de 25,4\% (Sexo masculino: 13,4\%. Sexofeminino: 11,9\%). De acordo com a faixa etária nos dois anos, a prevalência foi maior em adolescentes de 10 a 12 anos e representou $32 \%$

\footnotetext{
${ }^{1}$ Bacharel em Saúde Coletiva.

${ }^{2}$ Doutor em Fisiopatologia Clínica e docente da Universidade de Brasília.

3 Doutor em Saúde Pública e docente da Universidade de Brasília.

${ }^{4}$ Mestre em Epidemiologia e Consultor do Ministério da Saúde.

${ }^{5}$ Doutora em Ciências da Saúde e docente da Universidade de Brasília.

${ }^{6}$ Doutora em Ciências da Saúde e docente da Universidade de Brasília.
} 
Artigo original

Hegemonia - Revista Eletrônica do Programa de Mestrado em Direitos Humanos, Cidadania e Violência/Ciência Política do Centro Universitário Unieuro

ISSN: 1809-1261

UNIEURO, Brasília, número 23, Janeiro a Junho de 2018, pp. 78-93.

do total. Conclusão: Verificou-se elevada prevalência de excesso de peso nos escolares da Escola Classe 48 na cidade de Ceilândia - DF.

PALAVRAS-CHAVE:Prevalência. Sobrepeso. Obesidade. Crianças. Adolescentes.

ABSTRACT: Objective: To identify the anthropometric profile and the prevalence of obesity in children and adolescents from a public school in Ceilândia - DF in the period 2014 and 2015. Methods: Epidemiological cross-sectional study with a descriptive approach of anthropometric data of 531 students from afternoon School Class 48 to consider the following variables: state the anthropometric profile according to the year, sex and age. Results: The prevalence of obesity in the analyzed population was 34,5\% in 2014 (Male: 17,5\% Female: 17,1\%). In the year 2015 it was 25,4\% (Male: 13,4\% Female: 11,9\%). According to the age group in both years, the prevalence was higher in adolescents 10 to 12 years old and represented $32 \%$ of the total. Conclusion: There was a high prevalence of overweight in School Class 48 in the city of Ceilândia - DF.

KEYWORDS: Prevalence. Overweight. Obesity.Children.Teens.

Introdução

Desde o início de século XX, o Brasil vivencia uma situação de saúde que combina uma transição demográfica acelerada, transição epidemiológica singular e transição nutricional na qual expressa em uma presença fortemente hegemônica das doenças crônicas não transmissíveis (DCNT) sendo um desafio para a saúde pública. ${ }^{1}$

Segundo Mendes (2011), “os principais fatores determinantes para o aumento das doenças crônicas são: as alterações demográficas, mudanças nos padrões de consumo de alimentos e nos estilos de vida, a urbanização acelerada e as estratégias mercadológicas". ${ }^{2}$ No que diz respeito aos padrões de consumo e estilos de vida, a mudança do perfil nutricional que se desenha no Brasil revela a importância de um modelo de atenção à saúde que incorpore ações de promoção da saúde, prevenção e tratamento das DCNT. ${ }^{3}$

O excesso de peso que compreende como sobrepeso e obesidade começaram a despertar preocupação também durante o período da infância. No Brasil, a avaliação doestado nutricional de crianças de 5 a 9 anos de idade, estudada pela Pesquisa de 
Artigo original

Hegemonia - Revista Eletrônica do Programa de Mestrado em Direitos Humanos, Cidadania e Violência/Ciência Política do Centro Universitário Unieuro

ISSN: $1809-1261$

UNIEURO, Brasília, número 23, Janeiro a Junho de 2018, pp. 78-93.

Orçamentos Familiares (POF) 2008-2009, mostrou que o excesso de peso e a obesidade já atingem $33,5 \%$ e $14,3 \%$, respectivamente. ${ }^{4}$

Recentemente, a Organização Mundial da Saúde (OMS) relatou que o número global de crianças e adolescentes com obesidade e sobrepeso aumentaram de 32 milhões em 1990 para 42 milhões em 2013. Se as estimativas atuais continuarem, até 2025, o número de crianças com esse problema aumentará para 70 milhões em termos globais. ${ }^{5}$ Pegolo e Silva (2008) afirmam que uma das explicações para esse fenômeno no atual cenário são os fatores nutricionais inadequados decorrentes da transição nutricional. ${ }^{6}$

Por esse ângulo, o setor saúde em parceria com o setor educação tem por objetivo trazer melhoria na qualidade de vida dos escolares. Assim, no ano de 2007, foi criado o Programa Saúde na Escola (PSE), instituído pelo Decreto Presidencial n ${ }^{\circ}$ 6.286, vem contribuindo para o fortalecimento de ações no que diz respeito ao enfrentamento das vulnerabilidades que comprometem o pleno desenvolvimento dos escolares. ${ }^{7}$ A Política Nacional de Alimentação e Nutrição (PNAN), também assume o papel de estimular as ações intersetoriais, e de garantir a qualidade dos alimentos consumidos no país, promovendo práticas alimentares saudáveis, prevenindo e controlando os distúrbios nutricionais. ${ }^{8}$

Este estudo tem relevância para a Saúde Coletiva poisenvolve não apenas os aspectos nutricionais, como também cultural, econômico e reconhece o valor do acompanhamento do estado nutricional em escolares, através do Índice de Massa Corporal (IMC), priorizando o empenho por melhores condições de vida, relacionadas ao processo saúde-doença dos indivíduos e da coletividade.

Dessa forma, esta pesquisa tem por objetivo identificar o perfil antropométrico e a prevalência de sobrepeso e obesidade em crianças e adolescentes de uma escola pública da Ceilândia - DF no período de 2014 e 2015.

Métodos

Trata-se de um estudo descritivo com abordagem quantitativa do tipo transversal. Trata-se de um estudo descritivo com abordagem quantitativa do tipo transversal. O estudo foi realizado com 531 crianças e adolescentes da Escola Classe 48 localizada na regional da Ceilândia no Distrito Federal. A fonte das informações dos dados foi de origem primária, 
Artigo original

Hegemonia - Revista Eletrônica do Programa de Mestrado em Direitos Humanos, Cidadania e Violência/Ciência Política do Centro Universitário Unieuro

ISSN: 1809-1261

UNIEURO, Brasília, número 23, Janeiro a Junho de 2018, pp. 78-93.

coletados no período de 2014 e 2015. Os critérios de inclusão utilizados foram: escolares do período vespertino da escola analisada, do sexo masculino e feminino, do $1^{\circ}$ período ao $5^{\circ}$ ano, na faixa etária entre 5 e 12 anos.

A coleta dos dados antropométricos dos escolares aconteceu no período de Novembro de 2014 e Outubro de 2015. A coleta dos dados antropométricos, foi executada pelos pesquisadores e os alunos da disciplina de Epidemiologia Descritiva da Universidade de Brasília, devidamente treinados. Posteriormente para os cálculos dos índices antropométricos, foram utilizadas as médias das medidas coletadas.

\section{DESCRIÇÃO DA AVALIAÇÃO ANTROPOMÉTRICA}

Realizada por meio da antropometria que possui procedimentos de fácil entendimento e baixo custo além de ser um método pouco invasivo e isolado de diagnóstico nutricional de adultos e crianças. ${ }^{9}$ As medidas foram aferidas pelos alunos da turma de epidemiologia descritiva da Universidade de Brasília e pelas pesquisadoras.

Para a avaliação antropométrica foram coletados os seguintes dados dos escolares:

Peso:

Coletado em quilos $(\mathrm{Kg})$ por meio de uma balança digital do tipo Eletronic PersonalScale - Modelo EB6171 e com capacidade para 150 (Kg). Os integrantes da amostra foram posicionados com os pés centralizados, descalços, com o olhar para o horizonte, com o mínimo de roupa possível e sem adornos.

Estatura:

Coletada em metros (m) e realizada com um Estadiômetro portátil com 2,50 (m). Os integrantes foram orientados a ficarem em posição ereta, em pé, com os pés descalços e unidos, com os ombros e a parte posterior da cabeça posicionados adequadamente ao estadiômetro e os braços soltos ao longo do corpo e unidos, olhando sempre para frente. 
Artigo original

Hegemonia - Revista Eletrônica do Programa de Mestrado em Direitos Humanos, Cidadania e Violência/Ciência Política do Centro Universitário Unieuro

ISSN: 1809-1261

UNIEURO, Brasília, número 23, Janeiro a Junho de 2018, pp. 78-93.

Índice de Massa Corporal:

O IMC foi calculado pela relação entre o peso dividido pelo quadrado da altura do indivíduo, segundo as recomendações da Organização Mundial da Saúde (OMS, 2007) assim como expresso pela fórmula abaixo:

$$
\operatorname{IMC}=\frac{\text { Peso }(\mathrm{Kg})}{\text { Altura }(\mathrm{m})^{2}}
$$

Os pontos de corte e classificações do estado nutricional de crianças foram determinados a partir das curvas de referência de 2007 com valores de IMC em percentil, seguindo orientações para a coleta e análise de dados antropométricos em serviços de saúde, disponibilizadas pelo Ministério da Saúde e com base nos critérios estabelecidos pela OMS. ${ }^{10}$

\section{AVALIAÇÃO DOS DADOS}

Inicialmente, foi realizado um inquérito por meio de informações fornecidas pela direção escolar. O inquérito consistiu inicialmente em armazenar os dados de cada escolar e trouxe as seguintes informações: (1) Nome, (2) Sexo, (3) Idade, (4) Data de Nascimento e (5) Escolaridade. Em seguida, os pesquisadores realizaram o levantamento dos dados buscando as seguintes informações: (1) Peso, (2) Estatura, (3) IMC e (4) Classificação do IMC.

Para a avaliação dos dados antropométricos foi utilizado os pontos de corte e classificações do estado nutricional de crianças e adolescentes, a partir das curvas de referência de 2007 da OMS, disponibilizados pelo SISVAN WEB. Como critérios de diagnóstico foram utilizados os pontos de corte de índice de Massa Corporal (IMC) por faixa etária. 
Artigo original

Hegemonia - Revista Eletrônica do Programa de Mestrado em Direitos Humanos, Cidadania e Violência/Ciência Política do Centro Universitário Unieuro

ISSN: 1809-1261

UNIEURO, Brasília, número 23, Janeiro a Junho de 2018, pp. 78-93.

As variáveis de análise foram categorizadas da seguinte forma: (1) Perfil do estado antropométrico segundo o ano: 2014 e 2015. (2) Sexo: Masculino e Feminino. (3) Faixa Etária: 5 a 8 anos, 8 a 10 anos; 10 a 12 anos.

Posteriormente, os dados das etapas foram digitados e arquivados em planilha do programa Microsoft Excel, para análise estatística. Receberam tratamento estatístico descritivo e foram apresentados na forma de gráficos.

A presente pesquisa faz parte do projeto Avaliação do perfil antropométrico de estudantes na região administrativa de Ceilândia, no período de 2013 e foi aprovado pelo comitê de ética em Pesquisa da Faculdade de Ciências da Saúde da Universidade de Brasília (CEP/FS-UNB) de acordo com o parecer n 378.506.

Resultados

No ano de 2014, estavam matriculados no turno vespertino 354 alunos. Dentre esses, $74,3 \%$ (263) participaram da coleta dos dados antropométricos. Os resultados desses dados mostraram que mais da metade dos escolares $61,2 \%$ encontraram-se com o Índice de Massa Corporal (IMC) adequado. Entretanto, 91 (34,6\%) do total apresentaram excesso de peso, destacando 38 casos $(14,4 \%)$ de obesidade grave (Figura 1). 
Artigo original

Hegemonia - Revista Eletrônica do Programa de Mestrado em Direitos Humanos, Cidadania e Violência/Ciência Política do Centro Universitário Unieuro

ISSN: $1809-1261$

UNIEURO, Brasília, número 23, Janeiro a Junho de 2018, pp. 78-93.

Figura1 - Perfil antropométrico geral dos escolares da Escola Classe 48 no ano de 2014, Ceilândia/DF, $\mathrm{n}^{\circ}: 263$

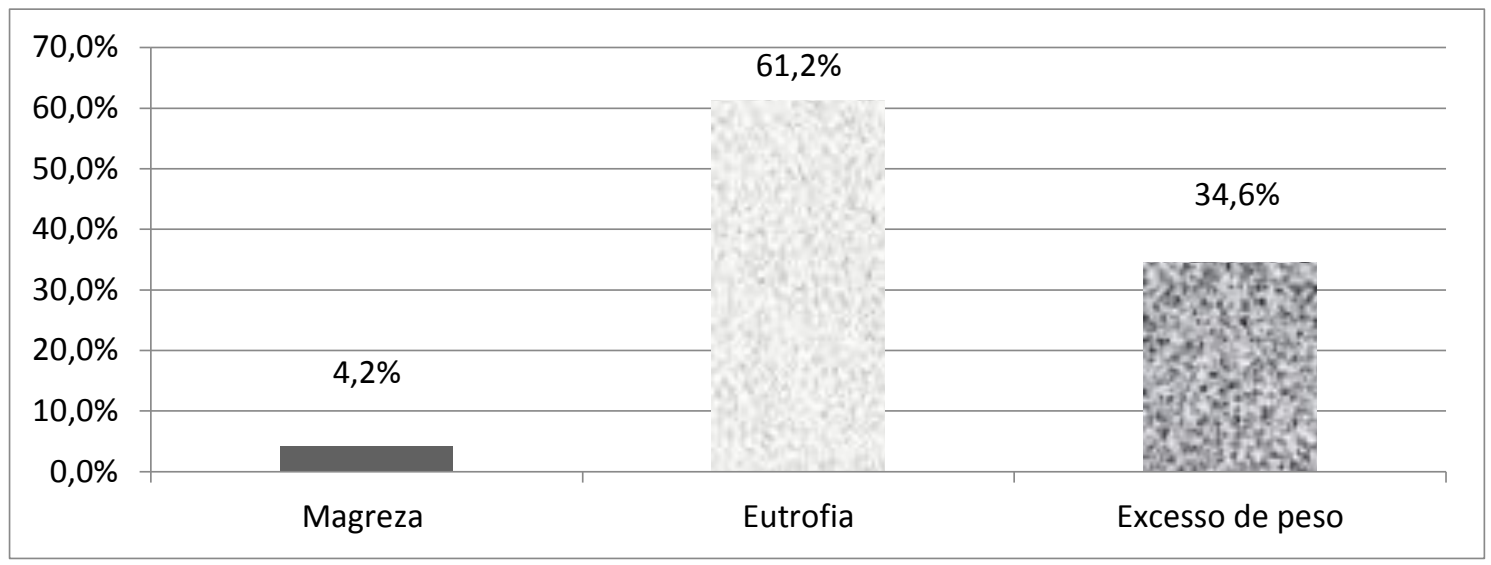

Em relação ao ano de 2015, dos 311 alunos matriculados no turno vespertino, 86,2\% (268) tiveram seus dados antropométricos coletados. Dentre esses, 194 (72,3\%) dos escolares estavam com o IMC adequado. Ademais, 68 (25,4\%) do total apresentaram excesso de peso, com maior prevalência para o sobrepeso representando 13,4\% com 36 casos (Figura 2).

Figura 2 - Perfil antropométrico geral dos escolares da Escola Classe 48 no ano de 2015, Ceilândia/DF, $\mathrm{n}^{\circ}: 268$

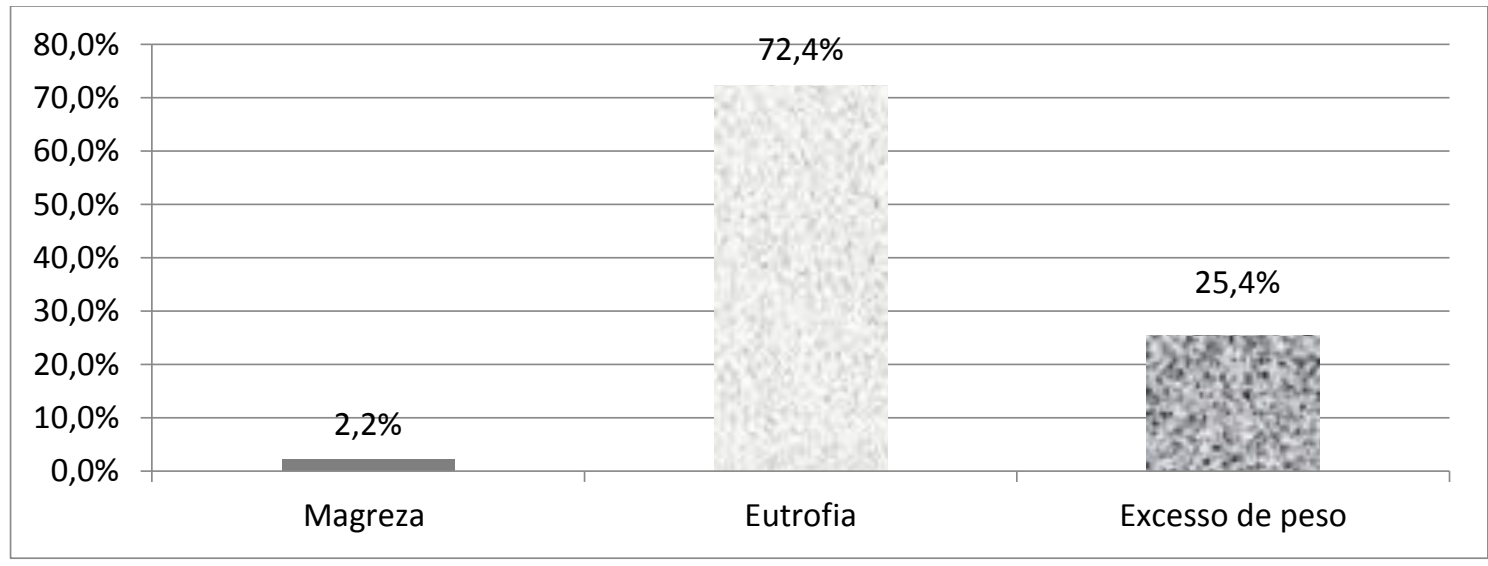

Quanto ao sexo, no ano de 2014, 137 (52\%) do total da amostra foi representada pelo sexo feminino. Já o masculino representou 126 (48\%). A análise dos dados 
Artigo original

Hegemonia - Revista Eletrônica do Programa de Mestrado em Direitos Humanos, Cidadania e Violência/Ciência Política do Centro Universitário Unieuro

ISSN: $1809-1261$

UNIEURO, Brasília, número 23, Janeiro a Junho de 2018, pp. 78-93.

antropométricos da Escola Classe 48, permitiu identificar excesso de peso em ambos os sexos. Em relação ao sobrepeso percebe-se que no sexo feminino houve 5,7\% e um predomínio no sexo masculino representando $8 \%$ com 21 casos. Já os valores referentes à obesidade foram maiores em meninas representando 11,4\% com maior predomínio de obesidade grave com 22 casos.

No ano de 2015 dos 268 escolares, $135(50,4 \%)$ da amostra foi composta pelo sexo feminino e 133 (49,6\%) pelo masculino. O sobrepeso foi prevalente no sexo masculino (7,5\%). Entretanto, comparando com os dados do ano de 2014, percebe-se uma pequena redução de $(0,5 \%)$ nos casos de sobrepeso no mesmo sexo. Já os casos de obesidade foram de $(5,9 \%)$ para ambos os sexos com 16 casos em cada um. Houve também redução nos casos de obesidade, principalmente a grave no sexo feminino que passou de 8,4\% no ano de 2014 para 2,6\% em 2015.

Verifica-se também poucos casos de magreza e magreza acentuada. Em 2014, o total foi de $4,2 \%$ do total dos casos. No ano de 2015 , houve redução de (2\%).

Ao verificar o IMC segundo a faixa etária de 5 a 8 anos, verifica-se um discreto predomínio de obesidade representando 18,4\% com 39 casos, em uma amostra de 212 crianças (Figura 3). Dentre esses achados, vale ressaltar que a obesidade foi maior em crianças de 5 anos de idade com 26 casos.

Figura 3 - Perfil antropométrico, segundo a faixa etária de 5 a 8 anos - 2014 e 2015,

$$
\text { Ceilândia/DF, } n^{\circ}: 212
$$

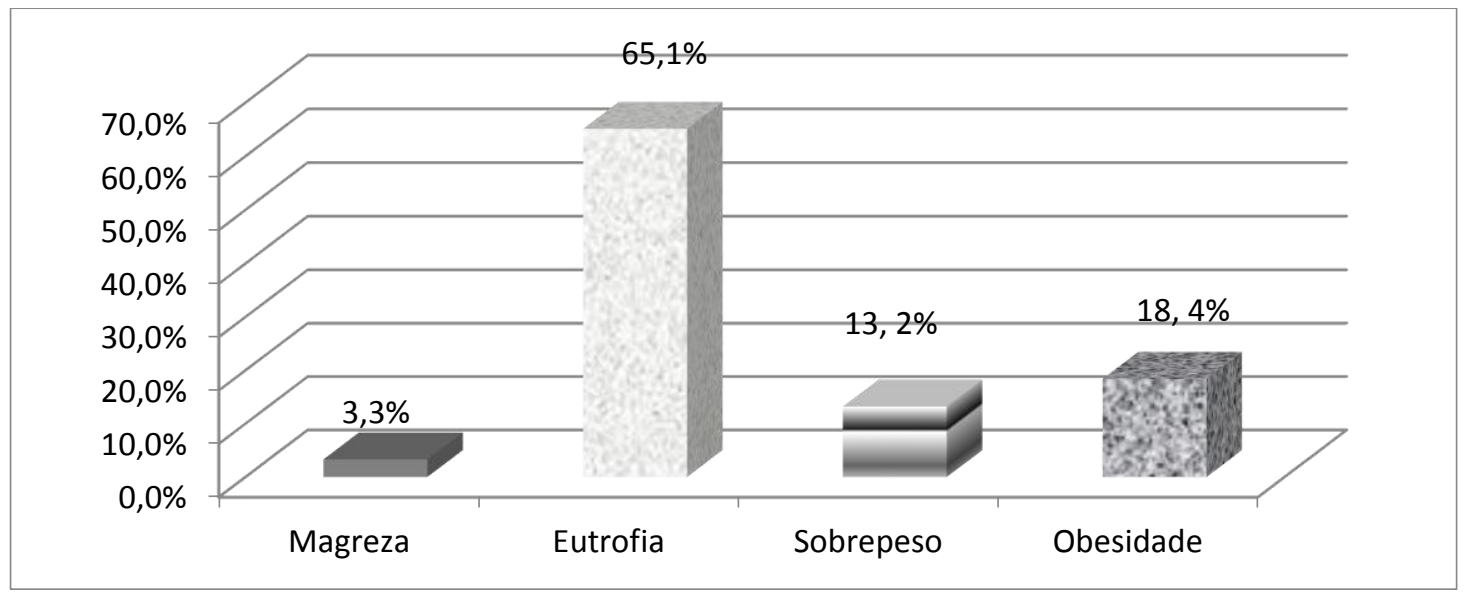


Artigo original

Hegemonia - Revista Eletrônica do Programa de Mestrado em Direitos Humanos, Cidadania e Violência/Ciência Política do Centro Universitário Unieuro

ISSN: $1809-1261$

UNIEURO, Brasília, número 23, Janeiro a Junho de 2018, pp. 78-93.

Já na faixa etária com crianças de 8 a10 anos, a prevalência de sobrepeso foi de 13,3\% e de obesidade 14,7\% com 33 casos em uma amostra de 225 crianças. Nessa faixa etária, os casos de magreza e de excesso de peso foram menores comparados as outras faixas etárias (Figura 4).

Figura 4 - Perfil antropométrico, segundo a faixa etária de 8 a 10 anos - 2014 e 2015,

Ceilândia/DF, nº: 225

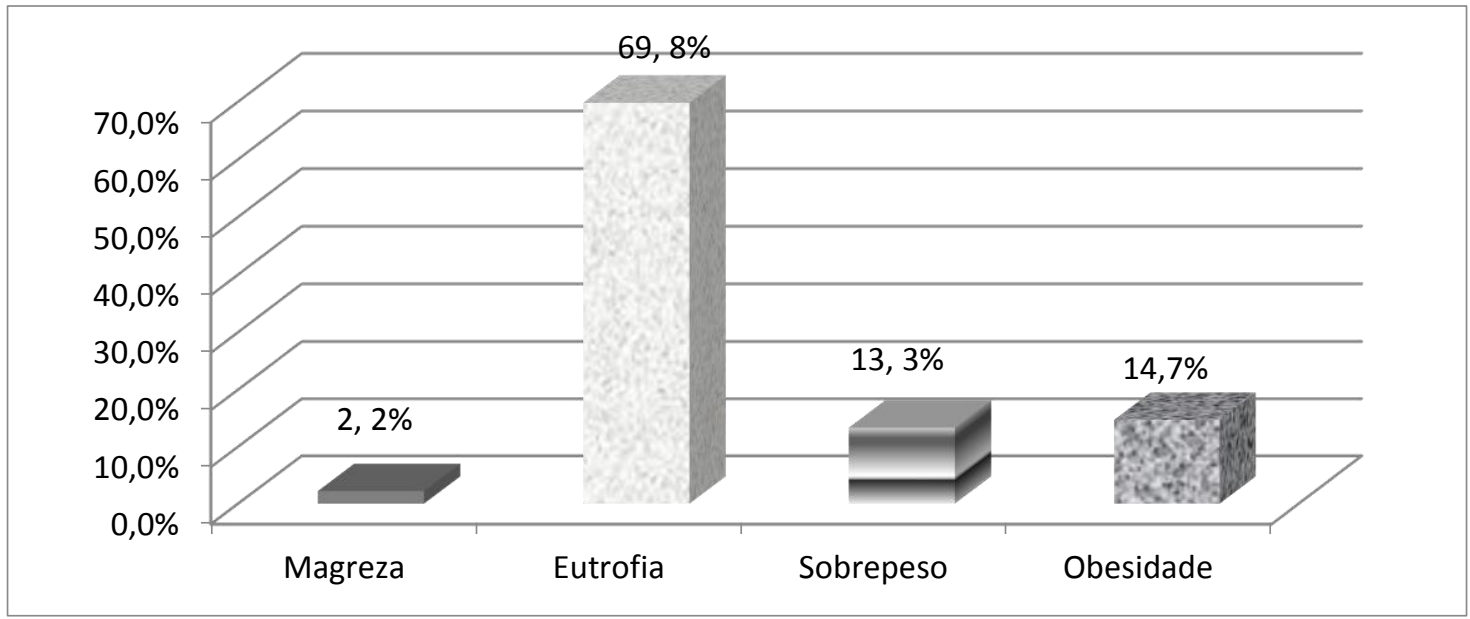

Em adolescentes de 10 a 12 anos, a prevalência de sobrepeso e obesidade foram iguais e representou (32\%) com 15 casos cada um em um total de 94 adolescentes. A magreza representou 4,2\% com poucos casos, assim como nas outras faixas etárias (Figura 5). 
Artigo original

Hegemonia - Revista Eletrônica do Programa de Mestrado em Direitos Humanos, Cidadania e Violência/Ciência Política do Centro Universitário Unieuro

ISSN: $1809-1261$

UNIEURO, Brasília, número 23, Janeiro a Junho de 2018, pp. 78-93.

Figura 5 - Perfil antropométrico, segundo a faixa etária de 10 a 12 anos - 2014 e 2015,

$$
\text { Ceilândia/DF, n: } 94
$$

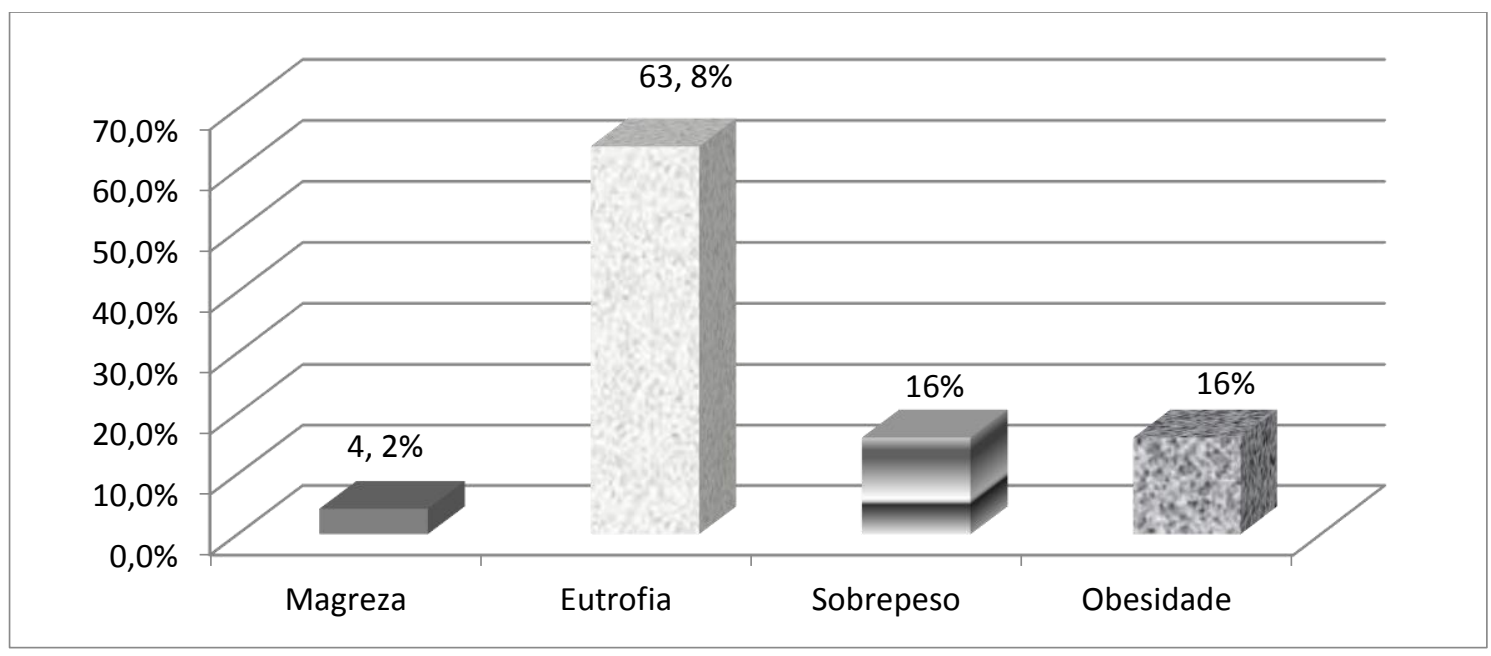

Discussão

Os dados obtidos no local do estudo mostram que o excesso de peso tem aumentado em crianças e adolescentes. Em 2014, na Regional de Saúde da Ceilândia, o sobrepeso foi de $13,29 \%$ e a obesidade $11,13 \%$ nas crianças de 5 a 10 anos em um total de 2.829 acompanhamentos registrados pelos serviços de saúde da cidade. Já nos adolescentes, o sobrepeso foi de $16,24 \%$ e a obesidade $6,84 \%$ em 3.306 acompanhamentos registrados. ${ }^{11}$

No ano de 2015, o sobrepeso foi de 14,45\% em crianças e 17,17\% em adolescentes em um total de 4.556 registros de acompanhamentos. ${ }^{11}$

Analisando os dados obtidos durante o período de 2014 e 2015 percebe-se que a maioria dos escolares encontra-se com o IMC adequado, porém, essa condição pode estar associada a uma redução na ingestão de alimentos tradicionais (como o arroz e o feijão) por produtos de baixo valor nutricional e alto conteúdo calórico como os industrializados (batata frita, hambúrguer, cachorro-quente, pizza, salgadinhos) ligados as atividades da rotina escolar, ou seja, cada vez mais crianças e adolescentes tendem a comer alimentos com calorias vazias, contudo ainda tem uma vida agitada na qual resulta no estado de eutrofia. ${ }^{12}$

Além disso, pode-se verificar o predomínio da alteração do excesso de peso entre escolares comparado com obesidade e obesidade grave. Esses resultados podem ser 
Artigo original

Hegemonia - Revista Eletrônica do Programa de Mestrado em Direitos Humanos, Cidadania e Violência/Ciência Política do Centro Universitário Unieuro

ISSN: 1809-1261

UNIEURO, Brasília, número 23, Janeiro a Junho de 2018, pp. 78-93.

comparados com o último estudo realizado pelo Instituto Brasileiro de Geografia e Estatística (IBGE) no ano de 2009 que mostra que o número de escolares com excesso de peso tem aumentado. Esse estudo também demonstrou que a obesidade e o sobrepeso foram os principais problemas nutricionais identificados em crianças e adolescentes nas cidades Brasileiras $^{13}$. Contudo, comparando os dados durante os dois anos de estudo, nota-se uma redução considerável de obesidade e obesidade grave (8,9\%) nos escolares.

É provável que essa redução durante o período seja o resultante das ações do Programa Saúde na Escola (PSE) que tem por objetivo a prevenção e o controle dos agravos nutricionais e de promoção da saúde de crianças e adolescentes. ${ }^{7}$ Ainda nesse contexto, Monteiro et al (2009) afirmam que "os principais fatores atribuíveis à redução deste agravo no Brasil se devem ao aumento da escolaridade materna, ao crescimento do poder aquisitivo das famílias, a expansão da assistência à saúde e a melhoria das condições de saneamento". ${ }^{14}$

Em relação a predominância de gênero, os resultados encontrados mostram disparidade com o estudo de Silveira (2015) onde o sobrepeso foi de 13,9\% nas meninas e $6,2 \%$ nos meninos. Contudo o estudo mostrou semelhança em relação a obesidade que foi maior no sexo feminino com $10,8 \%$ e $5,2 \%$ no sexo masculino. ${ }^{15}$

Da Silva (2002) afirma que "a partir dos 5 anos de idade, o excesso de peso e a obesidade são encontrados com grande frequência, em todos os grupos de renda e em todas as regiões brasileiras e pode ser causada por diversos fatores envolvendo questões biológicas, econômicas, sociais, culturais e políticas". ${ }^{16}$ Além disso, o aleitamento materno de maneira desordenada, o consumo de alimentos processados altamente calóricos precocemente, a diminuição do espaço para a prática de exercícios físicos e a incorporação de formas de lazer sedentárias, agravam esse cenário. ${ }^{17}$

Ainda de acordo com SD Castilho et al., (2014) " se o aumento da prevalência do excesso de peso não diminui com o estirão da puberdade, a população estará cada vez mais obesa e, consequentemente, com maior risco de ter sua saúde e produtividade comprometidas, trazendo um ônus cada vez maior à economia do país". ${ }^{17}$

Nessa perspectiva, alguns fatores podem estar atrelados ao excesso de peso nos escolares como os comportamentos sedentários, que estão relacionados à televisão, vídeo game e ao computador e também ligado a um padrão alimentar pouco saudável, relacionado ao consumo de alimentos industrializados e com baixa densidade nutricional. ${ }^{18}$. A diferença 
Artigo original

Hegemonia - Revista Eletrônica do Programa de Mestrado em Direitos Humanos, Cidadania e Violência/Ciência Política do Centro Universitário Unieuro

ISSN: 1809-1261

UNIEURO, Brasília, número 23, Janeiro a Junho de 2018, pp. 78-93.

de hábitos e condições sócio-econômicas das amostras nos dos dois anos pode ter influenciado na diferença dos resultados.

Com isso, a prevalência de excesso de peso neste estudo incluindo os dois anos foi de $30 \%$. Nesse sentido, pode-se se afirmar que esse aumento de excesso de peso é multifatorial admitindo-se que o seu desenvolvimento pode ser determinado por um ou pela soma de fatores genéticos, ambientais, comportamentais e socioculturais. ${ }^{19}$

Em relação aos países em desenvolvimento, a obesidade está presente nas diferentes faixas econômicas, principalmente nas faixas de classe mais alta resultando em padrões comportamentais específicos que afetam ingestão calórica, gasto energético e taxa de metabolismo. A obesidade também se faz presente em classes socioeconômicas menos favorecidas, uma vez que alimentos saudáveis estão menos disponíveis para indivíduos de condições mais restritas. ${ }^{20}$

Por outro lado, esse aumento pode está relacionado a uma alimentação rica em gorduras, sobretudo as de origem animal, açúcar e alimentos refinados e redução da ingestão de carboidratos complexos, micronutrientes e fibras, que favorecem o estoque energético provenientes da chamada dieta ocidental e associada também ao sedentarismo. ${ }^{21}$

Por esse ângulo, sabe-se que a alimentação não saudável é um dos fatores de risco para DCNT, contribuindo para a epidemia de sobrepeso e obesidade, pela elevada prevalência de hipertensão arterial e pelo colesterol alto. ${ }^{22}$

Assim, verifica-se então que a prevalência do excesso de peso entre os escolares acometeu todas as faixas etárias analisadas e tais resultados indicam a transição nutricional que já foi concluída nos países desenvolvidos, mas que ainda está se consolidando em países em desenvolvimento como o Brasil. Nessa perspectiva, observa-se um declínio dos casos de desnutrição em ritmo bem acelerado e aumento da prevalência de sobrepeso e obesidade. ${ }^{23}$

\section{Referências}

1. Mendes, Eugênio Vilaça. O cuidado das condições crônicas na atenção primária à saúde: o imperativo da consolidação da estratégia da saúde da família. Brasília: Organização PanAmericana da Saúde; 2012. p. 512 . 
Artigo original

Hegemonia - Revista Eletrônica do Programa de Mestrado em Direitos Humanos, Cidadania e Violência/Ciência Política do Centro Universitário Unieuro

ISSN: 1809-1261

UNIEURO, Brasília, número 23, Janeiro a Junho de 2018, pp. 78-93.

2. Mendes, Eugênio Vilaça. As redes de atenção à saúde. Brasília: Organização PanAmericana da Saúde; 2011. p. 549.

3. Ministério da Saúde (BR). Secretaria de Atenção à Saúde. Departamento de Atenção

Básica. Caderno de atenção básica: Obesidade. 1.ed. Brasília: Ministério da Saúde; 2006.

4. Instituto Brasileiro de Geografia e Estatística. Pesquisa de Orçamentos Familiares (POF) 2008-2009: Antropometria e estado nutricional de crianças, adolescentes e adultos no Brasil. Rio de Janeiro: Instituto Brasileiro de Geografia e Estatística; 2010.

5. Organização Mundial da Saúde. Organização Pan-Americana da Saúde. Doenças crônicas não transmissíveis: Estratégias de controle e desafios para o sistema de saúde. Genebra: Organização Mundial da Saúde; 2015.

6. Pegolo G, Silva M. Estado Nutricional de Escolares da Rede Pública de Ensino de Piedade, SP. Segurança Alimentar e Nutricional [Internet]. 2008 [Citado 2016 jun 30]; 15(1): 76-85. Disponível em: http://periodicos.sbu.unicamp.br/ojs/index.php/san/article/view/1826.

7. Ministério da Saúde (BR). Secretaria de Atenção à Saúde. Departamento de Atenção Básica. Instrutivo PSE. 1. ed. Brasília: Ministério da Saúde; 2011.

8. Ministério da Saúde (BR). Secretaria de Atenção à Saúde. Departamento de Atenção Básica. Política Nacional de Alimentação e Nutrição. 1. ed. Brasília: Ministério da Saúde; 2012.

9. Montarroyos, Ellen Christina Leinhardt; COSTA, Kelem Rodrigues Lima; FORTES, Renata Costa. Antropometria e sua importância na avaliação do estado nutricional de crianças escolares. Rev Ciência e Saúde. 2013; 24 (2):197-206.Disponível em:http://www.escs.edu.br/pesquisa/revista/2013Vol24_1_3_Antropometriaimportancia. pdf.

10. Ministério da Saúde (BR). Secretaria de Atenção à Saúde. Departamento de Atenção Básica. Orientações para a coleta e análise de dados antropométricos em serviços de saúde: Norma Técnica do Sistema de Vigilância Alimentar e Nutricional - SISVAN. 1. ed. Brasília: Ministério da Saúde; 2011.

11. Sistema de Vigilância Alimentar e Nutricional. Ministério da Saúde [Internet]. Brasília: Secretaria Executiva. Sisvanweb; 2015 [citado 2016 jun7] Disponível em: http://dabsistemas.saude.gov.br. 
Artigo original

Hegemonia - Revista Eletrônica do Programa de Mestrado em Direitos Humanos, Cidadania e Violência/Ciência Política do Centro Universitário Unieuro

ISSN: $1809-1261$

UNIEURO, Brasília, número 23, Janeiro a Junho de 2018, pp. 78-93.

12. Andrade, Roseli G.; Pereira, Rosangela A.; Sichieri, Rosely. Consumo alimentar de adolescentes com e sem sobrepeso do Município do Rio de Janeiro. Cad Saúde Pública [Internet]. 2003 set - out [ citado 2016 jul 8];20(4):93-107. Disponível em: http://www.scielo.br/pdf/\%0D/csp/v19n5/17821.pdf.

13. De Souza, Mariana Peres; Molz, Patrícia; Pereira, Camila Schreiner. Análise do consumo de alimentos fonte de sódio e excesso de peso em escolares do município de Rio Pardo, RS. RevCinergis. 2014; 15(1): 39-42.

14. Monteiro Carlos Augusto, Benicio Maria Helena D'Aquino, Konno Silvia Cristina, Silva Ana Carolina Feldenheimer da, Lima Ana Lucia Lovadinode, Conde Wolney Lisboa. Causas do declínio da desnutrição infantil no Brasil, 1996-2007. Rev Saúde Pública [Internet]. 2009 Fev [citado 2016 jul 30]; 43 (1): 35-43. Disponível em: http://www.scielo.br/scielo.php?script=sci_arttext\&pid=S003489102009000100005\&lng= en.

15. Silveira, Francisco José Ferreira da; Barbosa, Julia Carvalho; Vieira, Vanessa Aline Miranda. Prevalência de sobrepeso e obesidade em crianças de quatro escolas estaduais de Belo Horizonte, Minas Gerais. RevMéd Minas Gerais. 201525 (2): 180-186.

16. Da Silva, Cristiane Matos; Polubriaginof, Cláudia. Obesidade infantil: fatores de risco e intervenções de enfermagem pertinentes, 2012. RevEnferm UNISA. 2012; 13(2): 112-6.

17. Castilho SD, Nucci LB, Hansen LO, AssuinoSR.Prevalência de excesso de peso conforme a faixa etária em alunos de escolas de Campinas, SP. Rev paulpediatr. [Internet]. 2014 [citado 2016 jun7] 32(2):200-6. Disponível em: http://www.scielo.br/pdf/rpp/v32n2/pt_0103-0582-rpp-32-02-00200.pdf.

18. Hills Ap, King NA, Armstrong TP. The contribution of physical activity and sedentary behaviours to the growth and development of children and adolescents: implications for overweight and obesity. Sports Med. 2007; (37):533-45.

19. Jesus Gilmar M. de, Vieira Graciete O, Vieira Tatiana O, Martins Camila da Cruz, Mendes Carlos Maurício Cardeal, Castelão Elizia S.. Fatores determinantes do sobrepeso em crianças menores de 4 anos de idade. J Pediatr. (Rio J.) [Internet]. 2010 Ago[citado 2016 jun 09]; 86(4): 311-316. Disponível em: http://www.scielo.b r/scielo.php?script=sci_arttext\&pid=S002175572010000400011\&lng=en\&nrm=iso. 
Artigo original

Hegemonia - Revista Eletrônica do Programa de Mestrado em Direitos Humanos, Cidadania e Violência/Ciência Política do Centro Universitário Unieuro

ISSN: 1809-1261

UNIEURO, Brasília, número 23, Janeiro a Junho de 2018, pp. 78-93.

20. Palhares Dario. Obesidade infantil: como podemos ser eficazes?. JPediatr. (Rio J.) [Internet]. 2004 [citado 2016 jun 16]; 80 (5): 432-433. Disponível em: http://www.scielo.br/scielo.php?script=sci_arttext\&pid=S002175572004000400004\&lng= en\&nrm $=$ iso.

21. Valle Valéria Sales do, Biehl Cíntia, Mello Danielli Braga de, Fortes Marcos de Sá Rego, Dantas Estélio Henrique Martin. Efeito da dieta hipoenergética sobre a composição corporal e nível sérico lipídico de mulheres adultas com sobrepeso. Rev Nutr. [Internet]. 2010 dez [citado jun 09]; 23(6): 959-967. Disponível em: http://www.scielo.br/scielo.php?script=sci_arttext\&pid=S1415-527320100 $00600003 \& \operatorname{lng}=$ en\&nrm $=$ isso.

22. Malta Dc, Cezário AC, Moura L, Morais Neto OL,Silva Junior Jb. A construção da vigilância e prevenção das doenças crônicas não transmissíveis no contexto do Sistema Único de Saúde. EpidemiolServ Saúde [Internet]. 2006 jul-set [citado 2016 abr 10] 15(3): 47-65. Disponível em: http://scielo.iec.pa.gov.br/pdf/ess/v15n3/v15n3a06.pdf.

23. Ramires Elyssia Karine, Menezes Risia Cristina Egito de, Oliveira Juliana Souza, Oliveira Maria Alice Araújo, Temoteo Tatiane Leocádio, Longo-Silva Giovana et al . Estado nutricional de crianças e adolescentes de um município do semiárido do Nordeste brasileiro. Revpaulpediatr. [Internet]. 2014 Set [citado 2016 jun 9] ; 32( 3): 200-207. Disponível em: http://www.scielo.br/scielo.php?script=sci_artte $\mathrm{xt} \&$ pid $=\mathrm{S} 010305822014000300200 \& \operatorname{lng}=\mathrm{en} \& \mathrm{nrm}=$ isso. 